\title{
APORTE METODOLÓGICO: PROTOCOLO DE ACCESO A LA COMUNICACIÓN A DISTANCIA EN PERSONAS HOSPITALIZADAS EN TIEMPOS DE COVID-19
}

\author{
METHODOLOGICAL CONTRIBUTION: REMOTE COMMUNICATION ACCESS \\ PROTOCOL IN HOSPITALIZED PEOPLE IN TIMES OF COVID-19
}

\section{Felipe Roberto Ceballos Vargas ${ }^{1}$, Alexis Esteban Álvarez Toro ${ }^{2}$, Fabrizio Leandro Acevedo Pérez ${ }^{3}$, María Belén Herrera Moratorio ${ }^{4}$, Andrea Soledad Duarte Martínez ${ }^{5}$}

\begin{abstract}
RESUMEN
Actualmente, Chile atraviesa la crisis sanitaria más importante del último siglo debido a la pandemia de covid-19, por lo que la mayoría de los centros hospitalarios han tenido que modificar su funcionamiento habitual para dar respuesta a la contingencia. Uno de los cambios implementados que ha producido más impacto en la salud de las personas hospitalizadas ha sido la suspensión de las visitas, generando un estado de incertidumbre, soledad y ansiedad para usuarios y sus familiares. Es por esta razón, que surge el propósito de describir un aporte metodológico estructurado como un protocolo de atención clínica, desde una perspectiva de humanización de los cuidados sanitarios, que facilite la comunicación de las personas hospitalizadas con sus respectivas redes, utilizando los productos de apoyo tales como dispositivos audiovisuales y/o escritos, enmarcado dentro de una de las estrategias no farmacológicas aplicadas por el equipo de Terapia Ocupacional del Hospital Clínico San José.
\end{abstract}

PALABRAS CLAVES

Terapia Ocupacional; COVID-19; Paciente Internos; Humanización de la Atención.

1 Licenciado en Ciencias de la Ocupación Humana Universidad de Chile. Terapeuta Ocupacional Universidad de Chile.Terapeuta Ocupacional en el Hospital Clínico San José. ORCID: https://orcid.org/0000-0003-0160-5899. ResearcherID: AAH-9175-2021. 8271538, ceballos.to@ gmail.com, 985854847

2 Licenciado en Ciencias de la Ocupación Humana Universidad de Chile. Terapeuta Ocupacional Universidad de Chile. Terapeuta Ocupacional en el Hospital Clínico San José. ORCID: https://orcid.org/0000-0002-0689-3861 ResearcherID: AAH-8102-2021. Docente Clínico Universidad de Santiago de Chile. 8150000, alex.alvarez.to@gmail.com, 933702235

$3 \mathrm{Mg}$ @ Neurociencias Universidad de Chile Terapeuta Ocupacional Universidad de Chile. Terapeuta Ocupacional en el Hospital Clínico San José. Académico Universidad Nacional Andrés Bello. Académico Universidad de Santiago de Chile. ORCID: https://orcid.org/00000002-9455-3719 ResearcherID: AAH-8388-2021. 7770130, flacevedo@uchile.cl, 962463187

4 Terapeuta Ocupacional Universidad Mayor. Licenciada en Ciencias de la Ocupación Universidad Mayor. Terapeuta Ocupacional en el Hospital Clínico San José. ORCID: https://orcid.org/0000-0002-1129-3238 ResearcherID: AAH-8169-2021. 8320000, bherrera.to@gmail. com, 987494468

5 Licenciada en Ciencias de la Ocupación Humana Universidad de Chile. Terapeuta Ocupacional Universidad de Chile. Terapeuta Ocupacional en el Hospital Clínico San José. ORCID: https://orcid.org/0000-0003-2823-9841 ResearcherID: AAH-8141-2021. 8822438, andrea.sduartem@ gmail.com, 988933084 


\begin{abstract}
Chile is currently experiencing the highest healthcare crisis witnessed in the last century due to the covid-19 pandemic. As a response to this emergency, hospitals have implemented radical changes to their day-to-day operations and functions. One of the changes is the suspension of visits to inpatient facilities by their social support network ie. family and friends. This has significantly impacted the psychological and emotional health of patients, for example leading to uncertainty, loneliness and anxiety. As a response to this, it has been necessary to describe a methodological contribution as a clinical care protocol to facilitate communication between inpatients and social support network. The Occupational Therapy team at San José Clinical Hospital has employed the use assistive technologies such as audiovisual and/or written devices to do this. These technologies have enabled re-introduction of humanity into health care removed by the restrictions of the pandemic.
\end{abstract}

\title{
KEYWORDS
}

Occupational Therapy; COVID-19; Inpatients; Humanization of Assistance.

Recibido:15/01/2021

Aceptado: 31/05/2021 


\section{INTRODUCCIÓN}

La emergencia sanitaria por el covid-19 ha traído importantes consecuencias al funcionamiento del sistema de salud del país, el cual ha estado fuertemente condicionado por un aumento en la demanda de camas de hospitalización. En Chile se cuenta con 38.571 camas totales, de las cuales 640 corresponden a unidades de cuidados intensivos ( $\mathrm{UCl}$ ) y de acuerdo al compromiso de la autoridad sanitaria se alcanzaría una cifra de 43.000 camas totales y 1.400 camas UCl antes de alcanzar el auge de contagios en nuestro país (Arteaga, 2020). Debido a esto, ha existido la necesidad de reconvertir unidades de menor a mayor complejidad requiriendo la creación de protocolos de capacitación precoz, la incorporación de personal sanitario inexperto para facilitar el funcionamiento, y a su vez reemplazar al gran número de trabajadores contagiados en su labor y la reorganización de los mismos (Phua, et al., 2020). Por otro lado, las limitaciones socio-afectivas debido a la suspensión de visitas y uso de elementos de protección personal (EPP) en personas aisladas por confirmación o sospecha de CovID-19 han expuesto a las personas hospitalizadas a un mayor riesgo de desarrollar sentimientos de soledad, aislamiento, miedo, pérdida de identidad, intimidad y dignidad, sensación de dependencia, incertidumbre por falta de información, incomprensión y trastornos agudos de sus capacidades cognitivas, como el delirium (Heras La Calle, Cruz Martin, \& Nin , 2017).

Debido a todos los antecedentes previamente señalados, se ha hecho inevitable la sobrecarga laboral en el personal de salud, amenazando tanto la salud mental de los trabajadores como la de las personas hospitalizadas, dificultando aún más la calidad de la interacción y, por lo tanto, la entrega de una atención personalizada (Kang, Son, Chae, \& Corte, 2018). Desde la humanización de los cuidados se propone reflexionar en cuanto al aporte que entregan las redes de apoyo como familiares y cuidadores en términos de contención emocional, manejo de noticias difíciles, comodidad y bienestar; pues es muy probable que una vez pasada la etapa crítica de hospitalización dicho impacto se verá reflejado en el acceso y adherencia a estrategias de rehabilitación, realidad que podría empeorar la respuesta del sistema sanitario al no contar, oportunamente, con una directriz clara respecto al abordaje de las necesidades humanas previamente señaladas.
Descrito el contexto sanitario general, el equipo te Terapia Ocupacional (T.O) del Hospital Clínico San José (HCSJ) plantea la siguiente contribución metodológica para su aplicación en diversos contextos hospitalarios. Dicha propuesta, estructurada en un protocolo de atención clínica, describe una estrategia no farmacológica basada en el acceso a la comunicación e interacción entre las personas hospitalizadas en distintas unidades de atención y sus redes, a través de diversos productos de apoyo (PA).

Este trabajo facilitará la visualización de las necesidades previamente mencionadas ofreciendo una herramienta oportuna que promueva la humanización de los cuidados y prevenga la incidencia de complicaciones asociadas al aislamiento social dentro del contexto de atención clínica actual.

\section{MARCO TEÓRICO}

\section{Definiciones}

Delirium: síndrome que se manifiesta como un cambio agudo del estado mental. Se caracteriza por la falta de atención y alteración en la cognición, que se desarrolla durante un período corto de tiempo y con un curso fluctuante de síntomas (Fong, Davis, Growdon, Albuquerque \& Inouye, 2015).

Demencia: condición neurodegenerativa insidiosa. Se caracteriza por un deterioro cognitivo crónico y progresivo del nivel previo de rendimiento, en uno o más dominios cognitivos, que interfiere con la independencia en las actividades cotidianas (Fong, Davis, Growdon, Albuquerque \& Inouye, 2015).

Facilitador intercultural: profesional que facilita la comunicación entre el equipo de salud y la población migrante internacional, articulando las necesidades de los usuarios con la red de salud. Su foco es resolver las inequidades en salud que afectan a las personas migrantes (Sepúlveda \& Cabieses, 2019).

Sistemas aumentativos y alternativos de comunicación (SAAC): conjunto de recursos, sistemas o estrategias dirigidos a facilitar la comprensión y la expresión del lenguaje de personas que tienen dificultades en 
la adquisición del habla y/o en la escritura. Su finalidad es la de aumentar el habla o sustituirla (Montero González, 2003).

Producto de apoyo: Cualquier producto (incluyendo dispositivos, equipo, instrumentos, y software) fabricado especialmente o disponible en el mercado, usado por o para personas con discapacidad para su participación, para proteger, apoyar, entrenar, medir o sustituir estructuras y funciones corporales y actividades o para prevenir déficit, limitaciones en la actividad o restricciones en la participación (aenor. Comité Técnico de Normalización, 2016).

Ventilación mecánica invasiva (VMI): procedimiento de soporte ventilatorio que emplea un aparato mecánico para ayudar o sustituir la función ventilatoria, a través de un tubo orotraqueal (тот) o traqueostomía (TQT) (García Castillo, Rodríguez Serrano, Zamora García, \& Chicot Llano, 2014).

Ventilación mecánica no invasiva (VNI): cualquier modo de soporte ventilatorio que se proporciona sin el uso de un tubo endotraqueal o traqueotomía (Slutsky \& Ferguson, 2006).

\section{Humanización y Redes de apoyo}

El concepto de humanización en la atención en salud contempla establecer una visión particular de lo que significa ser humano, entendiendo a la persona hospitalizada como un sujeto de derechos y relevando formas de actuar sobre esta visión, tales como la sensibilidad y la ética en el acto del cuidado, resaltando la dignidad humana y ofreciendo una óptima atención, comunicación e información efectiva a los usuarios, sus familias y otros profesionales involucrados en el sistema de salud (Ariza Olarte, 2012) (Todres, Galvin, \& Holloway, 2009). Por lo tanto, el humanizar es buscar la excelencia abordando multidimensionalmente todas las facetas de una persona y no solamente el área clínica.

Teniendo en cuenta esta visión, la humanización en los cuidados es clave para considerar a la persona hospitalizada y familia como un binomio, el cual debe ser abordado integralmente y de forma activa en su estadía hospitalaria, llevando a cabo acciones específicas que promuevan este proceso como una dimensión transversal de la calidad de atención: horarios de visita, la comunicación efectiva, el bienestar de la persona hospitalizada, la participación de las familias en los cuidados, disminuir el síndrome de desgaste profesional y los cuidados al final de la vida (Alonso-Ovies \& Heras la Calle, 2019).

Por otro lado, la hospitalización de una persona provoca una alteración en la estructura familiar, enfrentando estresores emocionales, cognitivos y sociales, generando sentimientos de conmoción, incertidumbre, desesperanza, negación, enojo, culpa, ansiedad y miedo a la muerte de un miembro de la familia, considerando la situación como traumática (De la Cueva Ariza, et al., 2018). Por esto, es imprescindible considerar a la familia como un agente activo dentro del proceso de hospitalización, afirmando que al facilitar su contribución a la recuperación de la persona hospitalizada se pueden obtener efectos positivos no tan solo para el sujeto, sino también para ellos mismos, y los profesionales de la salud, reduciendo el estrés emocional y facilitando la cercanía y comunicación de las partes implicadas (Heras La Calle, Cruz Martin, \& Nin , 2017).

En el contexto de Unidades de paciente crítico (UPC) existen guías clínicas tales como las guías PADIS (Devlin, et al., 2018) usando al ABCDEF de Bundle como referencia, donde se considera el compromiso de la familia, incorporando en el plan de tratamiento sus necesidades e interrogantes como parte de un proceso de hospitalización y como un pilar que contribuye activamente en disminuir la ansiedad en la persona hospitalizada (Marra, Ely, Pandharipande, \& Patel, 2017). Asimismo, en diversos estudios se ha planteado que el rol de la familia y sus acciones son una de las estrategias no farmacológicas más utilizadas, por lo que el privarse de esta estrategia en contextos como el de pandemia por el CoviD-19 genera diversas consecuencias relacionadas directamente al bienestar de la persona hospitalizada y su red de apoyo.

En resumen, facilitar la humanización de los cuidados y la inclusión de las redes de apoyo, permite ampliar la mirada más allá de un enfoque clínico unidimensional, imprimiendo una atención sanitaria que identifique los factores precipitantes del desgaste y sufrimiento emocional y espiritual de las personas hospitalizadas y sus familias, para proporcionar una atención de alta calidad, más ética y humanizada. Por ende, la implementación de políticas que restringen las visitas familiares, debe ir seguida de esfuerzos adicionales para apoyar 
la interacción persona hospitalizada-familia. Esto debe incluir tiempo y esfuerzo dedicado para conversaciones telefónicas y de video durante el tiempo de hospitalización (Kotfis, et al., 2020).

\section{La comunicación a distancia como estrategia no farmacológica de intervención}

Existen estudios que sugieren que para los usuarios que se encuentran hospitalizados recibir visitas familiares disminuye su ansiedad, la confusión y la agitación, reduciendo además complicaciones cardiovasculares, estimulando de esta forma que se sientan más seguros y aumentando su satisfacción (Usher \& Hill, 2016). Sin embargo, los desafíos relacionados con la pandemia han dificultado la implementación de estrategias habituales en los hospitales públicos, como la participación presencial de los familiares. Por consiguiente, aspectos tales como el aislamiento social creado por el distanciamiento físico y la implementación de normas que restringen el ingreso de visitas a las unidades hospitalarias, han contribuido a la desconexión de las redes familiares generando sentimientos de soledad y sufrimiento social, tanto al usuario como la familia. Lo cual, ha facilitado un aumento de la aparición de estados o síndromes altamente prevalentes antes de la pandemia, como lo es el delirium (Kotfis, et al., 2020), siendo uno de los diagnósticos más frecuentes en personas hospitalizadas, especialmente en $\mathrm{UCl}$, con una incidencia cercana a un 80\% (Salluh, et al., 2010).

Además, este contexto de restricción ha facilitado la incidencia de síntomas psico conductuales como es en el caso de personas con demencia, cuyo escaso acceso a participación social ha afectado su estado de ánimo acentuando la presentación de problemas de comportamiento, lo cual no solo ha complicado el manejo de dichas personas, sino que también podría incrementar el costo de la atención médica y la necesidad de apoyo para quienes padecen de esta patología (Wang, et al., 2020).

A partir de lo anterior, se propone que, mediante la facilitación de estrategias no farmacológicas, permitidas y validadas en este contexto, como la comunicación a distancia multimodal entre las redes de apoyo y las personas hospitalizadas, se estarán aminorando las consecuencias del aislamiento social, disminuyendo así la expresión de potenciales factores de riesgo para la incidencia de síndromes agudos como el delirium. Por consiguiente, la promoción de este tipo de estrategias como un derecho fundamental de los usuarios de la red asistencial durante el estado de emergencia constituye un gran avance en la humanización de los cuidados hospitalarios, destacándose como una intervención primordial para una atención clínica de calidad y centrada en la persona.

\section{Productos de apoyo}

Si bien tradicionalmente el uso de las tecnologías asistivas (TA) en rehabilitación se ha asociado principalmente a la mejoría de la función corporal, durante los últimos años este enfoque ha cambiado hacia un enfoque orientado a la actividad y la participación (Ivanoff, Iwarsson, \& Sonn, 2006). Es por esta razón que según la norma técnica UNE-EN ISO 9999:2016 el uso de los PA, anteriormente conocidos como TA, constituyen un diverso y amplio grupo de recursos que promueven soluciones y tecnologías para mejorar la autonomía y el bienestar de las personas (Pousada, Pereira, Groba, \& Nieto, 2018). Cabe destacar el rol de los y las Terapeutas Ocupacionales (тт.0o) cuya interacción con los PA, se enmarca bajo una perspectiva integrada, en relación a las habilidades de la persona, su ocupación y el ambiente, lo que permite la comprensión de las barreras y los facilitadores para su acceso y adopción. (World Federation of Occupational Therapists, 2019).

Debido al contexto hospitalario en situación de pandemia, se considerarán los productos de apoyo para la comunicación y la información, definidos como elementos para ayudar a una persona a recibir, enviar, produciry/o procesar información de diferentes formas (aenor. Comité Técnico de Normalización, 2016), como una alternativa concreta y viable de comunicación a distancia para mantener el libre acceso a la comunicación y participación activa de estas personas en su proceso de hospitalización.

Finalmente, para facilitar la lectura en la propuesta metodológica, los PA en el protocolo de comunicación a distancia, serán mencionados por sus nombres comunes y no por la clasificación de la norma técnica UNE-EN ISO 9999:2016. Además, la selección del PA para facilitar la comunicación a distancia entre las personas hospitalizadas y sus redes de apoyo, no obedecerán a un modelo existente para la selección de PA. Por 
lo que se abordarán según criterios y consideraciones contextualizadas a la dinámica hospitalaria actual y criterios propios que plantea el equipo de T.O del HCSJ, entendiendo el limitado acceso a recursos tecnológicos y restricciones por la situación actual de pandemia de COVID-19.

\section{Protocolo de COMUNICACIÓN A DISTANCIA}

A continuación, se presenta la protocolización de una estrategia planteada por T.O para facilitar el acceso a la comunicación a distancia entre personas hospitalizadas con y sin diagnóstico de COVID-19 con sus redes de apoyo, situados en contexto de restricción de visitas.

\section{Objetivos}

\section{General}

- Favorecer la humanización de los cuidados sanitarios de la persona hospitalizada y sus redes de apoyo en el HCSJ.

\section{Específicos}

- $\quad$ Prevenir las consecuencias del aislamiento social y la privación del soporte emocional en personas hospitalizadas en el HCSJ, a través del uso de estrategias de comunicación a distancia con sus redes de apoyo.

- Promover estrategias de comunicación, contención y soporte emocional entre las personas hospitalizadas en el HCS y y sus redes de apoyo, a través del uso de dispositivos audiovisuales y/o escritos en el marco de la intervención de T.O.

- Considerar los factores de riesgo asociados a la hospitalización que conllevan a complicaciones frecuentes como el delirium y el síndrome post cuidados intensivos, a través de la estrategia no farmacológica de la comunicación a distancia en las dependencias del HCSJ.

\section{Alcance}

TT.OO que se desempeñen en unidades hospitalarias críticas, quirúrgicas y médicas del HCSJ.

\section{Responsabilidades}

Los y las TT.oo serán responsables de implementar estrategias de comunicación a distancia que promuevan un ambiente humanizado hacia las personas hospitalizadas. Este protocolo debe ser aprobado por él o la encargada de gestión del cuidado de cada servicio clínico donde se implementa y por los jefes de servicio.

\section{Población Objetivo}

La población objetivo de este protocolo son aquellas personas hospitalizadas en el HCS que se encuentren limitadas en su interacción con sus redes de apoyo y manifiesten el interés en establecer contacto con ellos en el marco de la atención directa de T.O, por lo que se debe incluir la indicación en ficha médica o interconsulta correspondiente.

\section{Mecanismo de acción}

La implementación de este protocolo contará con dos fases, las cuales se centrarán en facilitar el proceso de comunicación a distancia.

\subsection{Evaluación inicial}

En la fase de evaluación se tomarán en cuenta los factores de la persona hospitalizada, redes de apoyo y contextuales. Considerando facilitadores y limitantes en la aplicación del protocolo (ver tabla 1), los cuales serán abordados en los puntos 6 y 7 correspondientemente. 
TABLA 1 TABLA DE CRITERIOS Y CONSIDERACIONES PARA EL ACCESO A LA COMUNICACIÓN A DISTANCIA

\begin{tabular}{|c|c|c|}
\hline Persona Hospitalizada & Redes de Apoyo & Contexto \\
\hline $\begin{array}{l}\text { Capacidades } \\
\text { 1. Estado cuantitativo de la } \\
\text { conciencia. } \\
\text { 2. Estado cualitativo de la conciencia. } \\
\text { 3. Capacidad sensorial (visual o audi- } \\
\text { tiva principalmente). } \\
\text { 4. Lecto-escritura y grafomotricidad. } \\
\text { 5. Comunicación y lenguaje. } \\
\text { Elementos invasivos } \\
\text { 6. Soporte ventilatorio. } \\
\text { 7. Traqueostomía. } \\
\text { Factores personales } \\
\text { 8. Idioma. } \\
\text { 9. Interés y conocimiento de la estra- } \\
\text { tegia de comunicación. }\end{array}$ & $\begin{array}{l}\text { Calidad de red de apoyo } \\
\text { 1. Presencia de familiares o } \\
\text { red de apoyo operativas. } \\
\text { 2. Familiar o red de apoyo } \\
\text { responsable que consien- } \\
\text { te estrategia. } \\
\text { Acceso } \\
\text { tecnológico-comunicacional } \\
\text { 3. Recursos para la comu- } \\
\text { nicación y dominio en la } \\
\text { manipulación de canales } \\
\text { de comunicación. }\end{array}$ & $\begin{array}{l}\text { Recursos para la atención } \\
\text { 1. Conexión a red de internet. } \\
\text { 2. Disponibilidad de elementos de } \\
\text { protección personal (EPP) y acce- } \\
\text { sorios de protección para dispo- } \\
\text { sitivos audiovisuales. } \\
\text { 3. Productos de apoyo y aditamen- } \\
\text { tos para la intervención. } \\
\text { Valores y cultura organizacional } \\
\text { 4. Disposición del equipo de salud. } \\
\text { 5. Respeto a la confidencialidad y } \\
\text { privacidad durante intervención. } \\
\text { Condiciones ambientales } \\
\text { 6. Acústica ambiental. } \\
\text { 7. Luminosidad. }\end{array}$ \\
\hline
\end{tabular}

\subsection{Implementación de estrategias de Comunicación} De acuerdo a la evaluación e identificación de factores relacionados a la persona, la familia y el contexto, se determinarán las siguientes formas de aplicar la estrategia para la implementación de este protocolo:

\subsubsection{Uso de productos de apoyo para facilitar la} comunicación:

a) Tablets o celulares: la utilización de estos dispositivos podrá estructurarse bajo distintos formatos, dentro de los cuales se consideran: videollamadas (por medio de aplicaciones como WhatsApp (C), Skype (C) y Zoom (C), Ilamado por altavoz, mensaje de video (por medio de WhatsApp (C), mensaje de audio (por medio de WhatsApp (C) y correos electrónicos.

b) Cartas: enviadas por redes de apoyo a las personas hospitalizadas, principalmente a aquellos que no tienen acceso a internet o aparato tecnológico que permita una comunicación audiovisual. La carta debe ser entregada en el ingreso del hospital, con el nombre y habitación de la persona hospitalizada, para ser enviados como artículos personales. Debe existir coordinación previa entre la red de apoyo y personal. c) Pizarras u hojas blancas plastificadas: el mensaje escrito en la pizarra u hoja blanca plastificada puede ser enviado como formato imagen a familiares a través de tablet o celular.

d) Fotos: recepción de fotos de familiares o redes de apoyo a personas hospitalizadas que manifiesten su interés en recibirlas.

\section{Criterios de exclusión}

Se definirán criterios excluyentes y formas atingentes de comunicación dependiendo de cada caso.

- Estado cuantitativo de la conciencia: se considera como criterio excluyente que la persona hospitalizada no logre vigilia funcional, correspondiente a un puntaje menor a SAS 3 (Escala de sedación y agitación), correspondiente a una persona sedada, pero despierta al estímulo verbal o táctil suave. De la misma manera se excluyen personas con agitación psicomotora correspondientes a un puntaje mayor o igual a SAS 5, equivalente a una persona agitada, que logra tranquilizarse con instrucciones (Tobar, et al., 2008). 
- Estado cualitativo de la conciencia: se considera como criterio excluyente, aquellas personas hospitalizadas con puntaje menor a 3 puntos en la escala de cooperación Standardized five questions $\left(S_{5} Q\right)$. Un puntaje 3 en la escala $S_{5} Q$ se relaciona a un deterioro moderado en su cooperación (González Seguel, Arriagada Bravo, Lee Goic, \& Ugarte Ubiergo, 2017), criterio que se acuerda en el equipo como mínimo para facilitar la comunicación. En el caso específico de personas con sospecha o presencia de delirium se aplicarán pautas específicas; Confusion assessment method (CAM) o Confusion Assessment Method for the Intensive Care Unit (CAM-ICU) a modo de considerar la agitación psicomotora correspondiente de una persona con delirium hiperactivo, que no pueda focalizar su atención ni responder favorablemente ante la contención verbal.

\section{Consideraciones}

\subsection{CAPACIDAD Sensorial}

a. Déficit auditivo: si la persona hospitalizada presenta algún déficit sensorial auditivo, se le solicitará el PA correspondiente en buen estado. Si corresponde a un audífono, solicitar una pila de repuesto a su red de apoyo.

En el caso de no contar con PA para corregir el déficit sensorial auditivo, se procederá a entregar: pizarra junto a un plumón o lápiz y papel (solo si la persona hospitalizada tiene lecto escritura, abordada en punto 7.2)

- Estrategias de comunicación sugerida: videollamada, mensaje por video, correo electrónico, fotos y/o cartas.

b. Discapacidad auditiva: si la persona hospitalizada presenta alguna discapacidad auditiva se considerará si su lengua nativa corresponde al español o lengua de señas chilena ( $(\mathrm{SCH})$. Además, se tendrá en cuenta si logra lectura oro facial. Toda esta información será consultada con un familiar o redes de apoyo con las cuales la persona logre comunicarse efectiva y eficientemente. Cabe destacar que en el HCSJ no se cuenta con interprete de LSCH, por lo que en conjunto con su red de apoyo se buscará la mejor opción posible).
- Estrategias de comunicación sugerida: videollamada, mensaje porvideo (con subtítulos o en LSCH), correo electrónico, fotos y/o cartas.

c. Déficit visual: si la persona hospitalizada presenta algún déficit sensorial visual, se le solicitará a su red de apoyo el PA correspondiente, como lentes ópticos en buen estado con un estuche que los proteja. En el caso de no contar con PA para corregir el déficit sensorial visual, se considerarán exclusivamente las siguientes estrategias de comunicación:

- Estrategias de comunicación sugerida: videollamada, llamada telefónica, mensaje de video y mensaje de audio.

d. Discapacidad visual: si la persona hospitalizada presenta alguna discapacidad visual se favorecerá el uso exclusivo de las siguientes estrategias de comunicación

- Estrategias de comunicación sugerida: llamada telefónica y mensaje de audio.

\subsection{LECTOESCRITURA Y GRAFOMOTRICIDAD}

Se considerará la lecto-escritura y se consultará a la red de apoyo la presencia o ausencia de ésta, en casos donde la persona hospitalizada no logre comunicarse de forma verbal y pueda comunicarse de forma escrita. Se considerarán puntos críticos el: lograr prensión fina con el fin de tomar lápiz o plumón y el lograr escribir sus ideas a modo de comunicarlo en carta, pizarra y/o correo electrónico.

\subsection{Comunicación Y lenguaje}

En casos específicos de trastornos de la comunicación y del lenguaje se solicitará una evaluación fonoaudiológica previa, con el fin de objetivar capacidades de la persona hospitalizada y considerar atingencia de la comunicación con la familia o redes de apoyo.

Además, será evaluada en conjunto atingencia de SAAC de alta, mediana y/o baja tecnología (Ten Hoorn, Elbers, Girbes, \& Tuinman, 2016), tales como tableros de comunicación y ayudas de comunicación de salida de voz. 


\subsection{Elementos inVASivos}

a. VMI: considerando el impacto y la falta de habituación de un contexto crítico por parte de los familiares o redes de apoyo, serán consideradas las siguientes estrategias de comunicación como un flujo de información únicamente de la familia o redes de apoyo hacia la persona hospitalizada.

- Estrategias de comunicación sugerida: mensajes de audio, mensajes de video, correos electrónicos, fotos y cartas.

b. VNI: se favorecerá la comunicación en tiempos de ventana terapéutica de la vNı. En el caso de no ser posible la ventana terapéutica de la vNı el flujo de comunicación será exclusivamente de la familia o redes de apoyo hacia la persona hospitalizada, cuyas estrategias de comunicación serán las siguientes

- Estrategias de comunicación sugerida: mensajes de audio, mensajes de video, correos electrónicos, fotos y cartas

c. TQT: en los casos particulares que la persona hospitalizada esté sin VMI, pero mantenga una TQT, para facilitar la comunicación se considerará obligatoriamente que el sujeto haya sido evaluado por kinesiólogo y fonoaudiólogo donde se considerará el uso de válvulas de fonación u oclusión de la TQT O uso de SCAA.

\subsection{FACTORES PERSONALES}

a. Idioma: consultar a la persona hospitalizada y/o redes de apoyo el dominio de el o los idiomas con los cuales la persona se comunica con sus redes de apoyo, si es necesario, solicitar los servicios de un facilitador intercultural del hospital.

b. Interés y conocimiento de la estrategia de comunicación: dentro de los puntos críticos se considerará como alta prioridad de elección la estrategia de comunicación con la cual la persona hospitalizada refiere sentirse cómoda y/o logre manejar de forma independiente o semi independiente, considerando la cercanía ante la estrategia de comunicación en su vida cotidiana y conocimientos de elementos tecnológicos.

\subsection{CONSIDERACIONES DE LA RED DE APOYO}

a. Presencia y pertinencia de red de apoyo operativa: se evaluará la red de apoyo con la que cuenta la persona hospitalizada (cercanía, tipo de parentesco, entre otros aspectos). Además, se considerará el rol de un encargado de comunicación, el cual será responsable de la coordinación con el equipo profesional y concretar la estrategia de comunicación a utilizar, frecuencia y horarios en que se realizará.

b. Red de apoyo responsable que autoriza consentimiento: se contará con un familiar o red de apoyo (idealmente el o la encargado/a de comunicación) que será responsable de autorizar el consentimiento y aceptar las condiciones en que se realizará la comunicación, en caso de que la persona hospitalizada no pueda. De preferencia se contará con el consentimiento de la persona hospitalizada.

\subsection{Acceso tecnológico-comunicacional de la familia}

a. Recursos para la comunicación y dominio en la manipulación de canales de comunicación: para la evaluación de estos aspectos se confeccionó una lista de verificación (ver figura 1) el cual será aplicado a la red de apoyo de la persona hospitalizada al momento de la evaluación inicial: 


\section{FIGURA 1 LISTA DE VERIFICACIÓN DEL ACCESO TECNO- LÓGICO COMUNICACIONAL}

Acceso tecnologico comunicacional
$\square$ Red eléctrica
$\square$ Red de telefonía fija
$\square$ Red de telefonía móvil
$\square$ Internet por Wi-Fi
$\square$ Internet por Datos móviles
$\square$ Dispositivos de comunicación
$\square$ Computador con cámara web
$\square$ Computador sin cámara web
$\square$ Smartphone con cámara frontal
$\square$ Smartphone sin cámara frontal
$\square$ Tablet con cámara frontal
$\square$ Tablet sin cámara frontal
$\square$ Teléfono fijo
$\square$ Teléfono celular (no smarthphone)
$\square$ Otro:
$\square$ Dominio en canales de comunicación

audiovisuales y escritos
$\square$ Lecto-escritura
$\square$ Correo electrónico
$\square$ Whatsapp
$\square$ Zoom
$\square$ Skype
$\square$ Hangouts
$\square$ Otro:
$\square$

\subsection{CRITERIOS Y CONSIDERACIONES DEL CONTEXTO}

- Recursos para la atención

a. Conexión a red de internet: evaluar posibilidad de contar con red WiFi del hospital y/o de adquisición de tarjeta con datos móviles en el caso de videollamadas, mensajes unidireccionales por alguna aplicación que requiera internet.

b. Disponibilidad de EPP: en el caso que la persona hospitalizada se encuentre con alguna precaución de contacto, gotitas y/o aerosol, se debe contar con los EPP necesarios para el profesional a cargo, acorde a las normas institucionales de la unidad de infecciones asociadas a la atención en salud. En caso de no tener los EPP no se realizará la intervención.

c. Accesorios de protección para dispositivos audiovisuales: para el uso de dispositivos electrónicos tipo tablets se considerarán las precauciones de prevención de infecciones, los cuales se detallan a continuación:

I. Antes de cada uso, cada dispositivo electrónico será higienizado con alcohol al $70 \%$.

II. Durante su uso, se le pedirá a la persona hospitalizada que utilice guantes para su manipulación, los que serán desechados luego de su uso.

III. Al terminar el uso del dispositivo, se sanitizará nuevamente con alcohol al $70 \%$.

IV. Cada dispositivo contará con una bolsa plástica con cierre hermético, donde se depositará luego de ser sanitizada y permanecerá ahí entre cada uso.

d. PA y aditamentos para la intervención: para la aplicación de la estrategia de comunicación se tendrá en consideración la posición de la persona hospitalizada, favoreciendo la posición más segura y cómoda, por lo que se debe contar con el uso de PA como sillas de ruedas (si logra el sedente), una cama que permita mover el respaldo y/o Órtesis de posicionamiento si es que lo requiere.

\subsection{VALORES Y CULTURA ORGANIZACIONAL}

a. Disposición del equipo de salud: para este tópico se dará a conocer el protocolo a los encargados de las unidades en que se implementará esta estrategia; jefes de servicio, enfermera coordinadora y a los estamentos participantes del equipo de rehabilitación como kinesiólogos y fonoaudiólogos. Además, se contará con protocolo en redes sociales institucionales para conocimiento de la comunidad hospitalaria.

b. Respeto a la confidencialidad y privacidad durante intervención: en el marco de los derechos humanos se respetará la autonomía de la persona hospitalizada y la privacidad al momento de facilitar la comunicación, considerando elementos que se dispongan en cada habitación, tales como biombos o cortinas y elementos que pueda proporcionar las redes de apoyo como audífonos (propios de la persona hospitalizada).

\subsection{Condiciones AMBIENTALES}

a. Acústica ambiental: considerar el nivel de ruido del ambiente que pueda interferir en la comunicación. Evaluar horarios en que este sea menor e 
informar a los equipos de cada unidad que se realizará la estrategia de comunicación para disminuir las interrupciones.

b. Luminosidad: favorecer que el ambiente en donde se aplique la estrategia de comunicación cuente con luz natural o artificial que permitan una videollamada, lectura de cartas y/o redacción de correos electrónicos.

\section{Insumos}

Los insumos tienen directa relación con las posibilidades de acceso de cada persona hospitalizada y el nivel de asistencia que puedan presentar. Estos deberán ser otorgados por la red de apoyo o por el equipo de rehabilitación tratante, según sea el caso (ver insumos tabla 2).

TABLA 2 INSUMOS

\section{Insumos entregados por la red de apoyo}

Lentes ópticos con su funda o estuche correspondiente.

Productos de apoyo para facilitar la comunicación e información.

Audífonos para dispositivo tecnológico.

Pizarra blanca $35 \times 50 \mathrm{cms}$.

Insumos entregados por el equipo de rehabilitación

Plumón de pizarra.

Hojas blancas.

Hojas blancas plastificadas.

Lápiz pasta.

Bolsas con cierre hermético tamaño 20×30 o

$25 \times 35 \mathrm{cms}$.

Tablets. Cada dispositivo debe contar con sus accesorios, considerando cargador a corriente y audífonos.

Chips de número telefónico para aparatos que posean esta función.

Toalla de papel.

Desinfectante (alcohol 70\%).

\section{Discusión}

Si bien la comunicación entre personas hospitalizadas y sus redes de apoyo debería ser en forma presencial debido a que la comunicación efectiva depende, en gran parte, del lenguaje corporal (Pettinari \& Jessopp, 2001); el protocolo presentado acontece y se enmarca en el contexto sociosanitario particular por el que atraviesa el país, desde esa perspectiva surge la necesidad imperante de mantener no solamente el contacto comunicacional entre ambas partes, como una estrategia temporal, sino también la posibilidad de mantenerla como parte de la intervención habitual que realiza el equipo de TT.OO del HCS ante situaciones en que no exista o no se pueda efectuar comunicación presencial.

Frecuentemente, la praxis de la T.O desafía al paradigma hospitalario predominantemente biomédico; el cual enfatiza la etiología y tratamiento de las enfermedades, ya que visibiliza aspectos estructurales del sistema hospitalario relacionados con la cotidianidad de las personas, tales como percepciones, emociones, relatos y opiniones. Siendo el acceso a la comunicación interpersonal y aquellas actividades que favorecen el bienestar emocional, acciones concretas que habitualmente deberían ser implementadas bajo un trato humanizado y la facilitación de un ambiente emocional enriquecedor.

Este protocolo de comunicación a distancia se proyecta como una estrategia clara y viable de los y las тт.OO, quienes ejercen el rol de facilitadores de la comunicación promoviendo la disminución de las consecuencias del aislamiento social y síntomas depresivos, a modo de prevenir alteraciones cognitivas y emocionales de las personas hospitalizadas, y aún más importante, el relevar la humanización en los cuidados sanitarios al promover el rol de las redes de apoyo como un ente activo en los procesos de salud. Es por esto que se plantea como desafío conocer el grado de satisfacción de las personas hospitalizadas y sus redes de apoyo quienes hayan sido partícipes de esta nueva estrategia y la percepción de los equipos de cada unidad donde se aplique dicha intervención.

Ciertos proyectos tales como el de Humanización en las Unidades de Cuidados Intensivos (Baeza Gómez \& Quispe Hoxsas, 2020), dentro de sus líneas estratégicas plantean la inclusión de los familiares facilitando su accesibilidad, presencia y participación de los procedimientos y soporte según las necesidades emocionales 
y psicológicas de estos y el sujeto en particular. (Grupo de trabajo de certificación de Proyecto $\mathrm{HU}-\mathrm{Cl}, 2 \mathrm{2019}$ ). Nuestro sistema de salud debe sumarse a esta nueva forma de entender los cuidados sanitarios y ciertas acciones como las que se plantean en este protocolo de comunicación a distancia se acercan y ajustan a este tipo de líneas estratégicas humanizadoras.

Cabe destacar que el uso de los PA ha sido un punto clave en la formulación de este protocolo de comunicación a distancia, por lo que considerar a futuro utilizar la norma técnica UNE-EN ISO 9999:2016 facilitará su entendimiento en dispositivos sanitarios de países de habla hispana. Se espera incluir progresivamente la norma técnica antes mencionada, según el contexto hospitalario se habitúe a la nueva estrategia metodológica y su nomenclatura.

Se busca proyectar la utilización de PA para la comunicación a distancia de manera protocolizada como una estrategia altamente aplicable, no obstante, la implementación podría constituir un gran desafío dada una gran diversidad de recursos humanos y contextuales en los distintos prestadores de servicios clínicos. Una de las limitaciones en esta propuesta metodológica situada en el HCSj es la escasez de recursos monetarios para el acceso a PA, tanto por el sistema sanitario como por las redes de apoyo de los sujetos descritos. Por lo tanto, la selección de los PA deriva del razonamiento clínico situado al contexto hospitalario por sobre la aplicación de un modelo de selección de PA, cuyo espectro de posibilidades está fuera del alcance práctico actual de la disciplina en el HCS en situación de pandemia. Sin embargo, se recomienda y proyecta la utilización de modelos para selección de PA, tales como el Modelo Human Activity Assistive Technology (HAAT) (Giesbrecht, 2013) a medida que avance la implementación y reformulación de las estrategias vertidas en este artículo en el HCSJ.

En conclusión, la emergencia sanitaria por CoviD-19 ha conducido a un cambio estructural del sistema de salud público y privado. Parte de estos cambios incluyen la restricción de las visitas a las personas hospitalizadas, por lo que, surge la comunicación a distancia como una estrategia no farmacológica viable en un hospital público y que se enmarca en la humanización de los cuidados sanitarios al restablecer el acceso a la comunicación con las redes de apoyo durante el proceso de hospitalización. De esta manera, se amplía el ejercicio clínico de la T.O planteando esta estrategia, hacia la visualización y resolución de necesidades que son rápidamente omitidas producto de la precarización de la atención durante una emergencia sanitaria.

La presente investigación no ha recibido ayudas específicas provenientes de agencias del sector público, sector comercial o entidades sin ánimo de lucro.

\section{AgradeCIMIENTOS}

Queremos agradecer a T.O Paulina del Solar, T.O Alejandra Ávila, T.O Miguel Fuentes, T.O Michelle Socias, T.O Bárbara Peralta, T.O Ximena Fuentes, T.O Francisco Bustos, T.O Eduardo Romero, Fgo. Benjamín Calixto, Dr. Dante Alberti y Dra. Carolina González quienes contribuyeron de forma valiosa en la confección y revisión de este protocolo de comunicación a distancia.

\section{RefERENCIAS BibLIOGRÁfICAS}

AENOR. Comité Técnico de Normalización. (2016). Productos de apoyo para personas con discapacidad. Clasificación y terminología. (ISO 9999:2016). Madrid: Asociasión Española de normalización.

Alonso-Ovies, A., \& Heras la Calle, G. (2019). Dígame qué necesita. Le escucho. Medicina Intensiva, 43(4), 193-194. doi:10.1016/j. medin.2018.09.003.

Ariza Olarte, C. (2012). Soluciones de humanización en salud en la práctica diaria. Enfermería Universitaria, 9(1), 41-51. doi:10.22201/ eneo.23958421e.2012.1.245

Arteaga, Ó. (2020). COVID-19. Revista médica de Chile, 148(3). doi:10.4067/ S0034-98872020000300279

Baeza Gómez, I., \& Quispe Hoxsas, L. C. (2020). Proyecto “Humanizando los Cuidados Intensivos", nuevo paradigma de orientación de los Cuidados Intensivos. Revista de Bioética y Derecho, 48, 111-126. doi:10.1344/rbd2020.48.29173

De la Cueva Ariza, L., Delgado Hito, P., Martinez Estalella, G., Via Clavero, G., Luch Canut, T., \& Romero García, M. (2018). Implementation of the evidence for the improvement of nursing care to the critical patient's family: a Participatory Action Research. BMC health services research, 18(1), 357. doi:10.1186/s12913-018-3177-8

Devlin, J., Skrobik, Y., Gélinas, C., Needham, D., Slooter, A., Pandharipande, P., ... Alhazzani, W. (2018). Executive Summary: Clinical Practice Guidelines for the Prevention and Management of Pain, Agitation/Sedation, Delirium, Immobility, and Sleep Disruption in Adult Patients in the ICU. Critical Care Medicine, 46(9), 15321548. doi:10.1097/CCM.0000000000003259 
Fong, T., Davis, D., Growdon, M., Albuquerque, A., \& Inouye, S. (2015). The Interface of Delirium and Dementia in Older Persons. The Lancet. Neurology, 8, 823-832. doi:10.1016/S1474-4422(15)00101-5

García Castillo, E., Rodríguez Serrano, D. A., Zamora García, E., \& Chicot Llano, M. (2014). Ventilación mecánica no invasiva e invasiva. Medicine, 17(63), 3759-3767. doi:10.1016/S0304-5412(14)70840-6

Giesbrecht, E. (2013). Application of the Human Activity Assistive Technology model for occupational therapy research. Australian Occupational therapy journal, 60(4), 230-240. doi:10.1111/14401630.12054

González Seguel, F. A., Arriagada Bravo, A. A., Lee Goic, J. E., \& Ugarte Ubiergo, S. (2017). Feasibility and Clinical Utility of ICF Framework in Critical ill Patients: Case Report. Annals of Musculoskeletal Disorders, 7(1), 1002.

Grupo de trabajo de certificación de Proyecto HU-Cl. (2019). Manual de buenas prácticas de humanización en Unidades de Cuidados Intensivos. Obtenido de http://humanizandoloscuidadosintensivos.com/es/buenas-practicas/

Heras La Calle, G., Cruz Martin, M., \& Nin , N. (2017). Seeking to humanize intensive care. Revista Brasileira de Terapia Intensiva, 29(1), 9-13. doi:10.5935/0103-507X.20170003.

Ivanoff, S. D., Iwarsson, S., \& Sonn, U. (2006). Occupational therapy research on assistive technology and physical environmental issues: a literature review. Canadian journal ofoccupational therapy, 73(2), 109-119. doi:10.1177/000841740607300203

Kang, H. S., Son, Y. D., Chae, S.-M., \& Corte, C. (2018). Working experiences of nurses during the Middle East respiratory syndrome outbreak. International journal of nursing practice, 5, e12664. doi:10.1111/ijn.12664

Kotfis, K., Williams Roberson, S., Wilson, J. E., Dabrowski, W., Pun, B. T., \& Ely, E. W. (2020). COVID-19: ICU delirium management during SARS-CoV-2 pandemic. Critical care, 24(1), 176. doi:10.1186/ s13054-020-02882-x

Marra, A., Ely, E. W., Pandharipande, P., \& Patel, M. B. (2017). The ABCDEF Bundle in Critical Care. Critical care clinics, 33(2), 225-243. doi:10.1016/j.ccc.2016.12.005

Montero González, P. (2003). Sistemas alternativos y aumentativos de comunicación (SAAC) y accesibilidad. Puertas a la lectura, 4, 129-136.

Pettinari, C. J., \& Jessopp, L. (2001). "Your ears become your eyes": managing the absence of visibility in NHS Direct. Journal of Advanced nursing, 36(5), 668-675. doi:10.1046/j.1365-2648.2001.02031.x

Phua, J., Weng, L., Ling, L., Egi, M., Lim, C.-M., Divatia, J. V., .. D Du, B. (2020). Intensive care management of coronavirus disease 2019 (COVID-19): challenges and recommendations. The Lancet. Respiratorymedicine, 8(5), 506-517. doi:10.1016/S2213-2600(20)30161-2

Pousada, T., Pereira, J., Groba, B., \& Nieto, L. (2018). Valorando la efectividad e impacto de los productos de apoyo en la vida de las personas con diversidad. La importancia de la medida de resultados. XJornadas Científicas Internacionales de Investigación sobre Personas con Discapacidad. Libro de Actas en CD.
Salluh, J. I., Soares, M., Teles, J. M., Ceraso, D., Raimondi, N., Nava, V. S.,... Rocha, M. G. (2010). Delirium epidemiology in critical care (DECCA): an international study. Critical Care, 14(6), 210. doi:10.1186/cc9333

Sepúlveda, C., \& Cabieses, B. (2019). Rol del Facilitador Intercultural para migrantes internacionales en centros de salud chilenos: perspectivas de cuatro grupos de actores clave. Revista Peruana de Medicina Experimental y Salud Publica, 36(4), 592-600. doi:10.17843/rpmesp.2019.364.4683

Slutsky, A. S., \& Ferguson, N. D. (2006). Noninvasive Ventilation. Encyclopedia of Respiratory Medicine, 455-459. doi:10.1016/B012-370879-6/00428-2

Ten Hoorn, S., Elbers, P. W., Girbes, A. R., \& Tuinman, P. R. (2016). Communicating with conscious and mechanically ventilated critically ill patients: a systematic review. Critical care (London, England), 20(1), 333. doi:10.1186/s13054-016-1483-2

Tobar, E., Lanas, A., Pino, S., Aspeé, P., Rivas, S., Prat, D., ... Castro, J. (2008). Sedación guiada por protocolo versus manejo convencional en pacientes críticos en ventilación mecánica. Revista médica de Chile, 136(6), 711-718. doi:10.4067/S0034-98872008000600004

Todres, L., Galvin, K., \& Holloway, I. (2009). The humanization of healthcare: A value framework for qualitative. International Journal of Qualitative Studies on Health and Well-being, 4(2), 68-77. doi:10.1080/17482620802646204

Usher, B., \& Hill, K. (2016). Family Visitation in the Adult Intensive Care Unit. Critical care nurse, 36(1), 15-8. doi:10.4037/ccn2016677

Wang, H., Li, T., Barbarino, P., Gauthier, S., Brodaty, H., Molinuevo, J. L., ... Yu, X. (2020). Dementia care during COVID-19. Lancet (London, England), 10231, 1190-1191. doi:10.1016/S0140-6736(20)30755-8

World Federation of Occupational Therapists. (2019, Marzo). Occupational Therapy and Assistive Technology. Retrieved from https:// www.wfot.org/resources/occupational-therapy-and-assistivetechnology 